\title{
Kernos
}

Revue internationale et pluridisciplinaire de religion grecque antique

4 | 1991

Varia

\section{Recherches scientifiques du C.E.R.G.A.}

Ioannis Loucas et Éveline Loucas-Durie

\section{OpenEdition \\ Journals}

\section{Édition électronique}

URL : http://journals.openedition.org/kernos/314

DOI : $10.4000 /$ kernos.314

ISSN : 2034-7871

\section{Éditeur}

Centre international d'étude de la religion grecque antique

\section{Édition imprimée}

Date de publication : 1 janvier 1991

Pagination : 317-322

ISSN : 0776-3824

\section{Référence électronique}

Ioannis Loucas et Éveline Loucas-Durie, «Recherches scientifiques du C.E.R.G.A. », Kernos [En ligne], 4 | 1991, mis en ligne le 11 mars 2011, consulté le 16 septembre 2020. URL : http:// journals.openedition.org/kernos/314 
Kernos, 4 (1991), p. 317-322.

\section{RECHERCHES SCIENTIFIQUES DU C.E.R.G.A.}

Dans le but de reprendre les recherches archéologiques du site arcadien de Lykosoura (suspendues depuis la fin du XIXe s.), le Comité de Recherches scientifiques du C.E.R.G.A., en coopération avec l'Éphore des Antiquités de Laconie et d'Arcadie, le prof. Th. Spyropoulos, a établi le plan de travail suivant :

1. Étude et publication des objets en terre cuite conservés au musée local dont une petite partie seulement figure dans l'ancien catalogue de Kourouniotis (ouvrage édité en 1911 et aujourd'hui épuisé).

2. Étude et publication des objets en terre cuite provenant du site de Lykosoura conservés dans les dépôts du Musée archéologique national d'Athènes et qui comptent notamment des statuettes zoocéphales.

3. Localisation des membres architecturaux provenant des édifices du sanctuaire (temple et mégaron de Despoina, portiques, etc.), réemployés lors de la construction ou de la réfection d'édifices modernes de la région (principalement des petites chapelles rupestres).

4. Prospection archéologique de surface.

5. Étude topographique de la région afin de situer l'oracle de Pan et les autres sanctuaires que Pausanias a vus dans la région (VIII, 37, 1012) mais qui n'ont pas été mis au jour par les fouilles de Cavvadias, de Léonardos et de Kourouniotis, ni par les sondages plus récents limités aux alentours du temple de Despoina.

6. Fouilles systématiques afin de découvrir les lieux évoqués cidessus.

Pendant la période 1989-1990, une équipe de recherche du C.E.R.G.A., composée de deux de ses administrateurs, Ioannis Loucas et Éveline Loucas-Durie, en coopération avec l'Éphore Th. Spyropoulos, a entamé l'exécution des points 1,3 et 4 du plan ci-dessus. L'étude du matériel étant en cours, voici un bref rapport des activités réalisées :

\section{A) Étude des objets en terre cuite du musée de Lykosoura}

Tous les objets ont été photographiés, mesurés et examinés sur place. Les couleurs ont été enregistrées avec leur dénomination commune et simultanément codifiées sur base du Code Munsell. Le répertoire constitué comprend : 1) 38 statuettes de figures humaines, divines ou animales, entières et fragmentaires (fig. 1). 2) 36 plaques votives 
portant des représentations de caducées (kerykeia) de divers types (fig. 3). 3) Deux lampes (fig. 2), dont l'une porte la signature du céramiste Spôsianos (IIe s. ap. J.-C.). 4) 1 skyphos miniature. 5) 3 cylindres creux servant vraisemblablement à des libations offertes à la déesse chthonienne du sanctuaire (fig. 4). 6) 8 tessons de céramique couvrant l'ère qui s'étend depuis les temps géométriques jusqu'à l'époque romaine. 7) 11 poids de tisserand. 8) 3 fragments de tuile, dont deux sont estampés.

L'étude des plaques votives à caducée ayant été achevée en octobre 1990, la publication totale du matériel est prévue pour la fin 1991 ou le début 1992.

\section{B) Recherches sur le terrain}

Elles ont été effectuées dans l'aire délimitée par une ligne discontinue sur la carte topographique (fig. 5).

I. Localisation des membres architecturaux provenant des édifices du site antique

a) Chapelle de Aghios Georghios (Aï-Ghiorghis), construite sur la colline qui constituait l'acropole - et la ville ? - antique : l'autel de la chapelle est formé d'un chapiteau dorique bien conservé, placé sur un autre abîmé, le tout reposant sur deux tambours (h. totale : $94 \mathrm{~cm}$ ); le pupitre est constitué d'un fragment de colonne dorique $(\mathrm{h} .: 77 \mathrm{~cm}$, diam. : $30 \mathrm{~cm}$ ); devant l'entrée de la chapelle gît une partie de colonnette (h. : $51 \mathrm{~cm} \mathrm{d.} \mathrm{:} 30 \mathrm{~cm}$ ); à $c .5 \mathrm{~m}$. du mur sud se trouve une grosse plaque en marbre $(128 \times 73 \times 20 \mathrm{~cm})$; à c. $20 \mathrm{~m}$. au sud-est de la chapelle a été repérée la partie supérieure d'un pilier (h. : $36 \mathrm{~cm})$.

b) Chapelle de Prophitis Elias (dite aussi Aï Ilia), sur la colline au nord-est de la colline du sanctuaire de Despoina : devant la porte de la chapelle git un fragment de colonnette et sur le jambage de la fenêtre de son mur sud a été vérifiée l'existence de la nouvelle loi sacrée repérée par A. Matthaiou et Y. Piloukas en 1986 (Horos, 4, p. 75-78, pl. 10-12), dont nous reproduisons ici le texte:

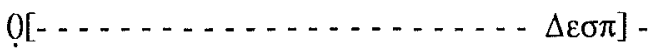

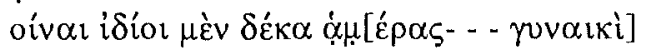

$3 \delta \dot{\varepsilon} \lambda \varepsilon \chi \circ \hat{\imath} \alpha \ddot{n} \pi \theta_{\iota} \dot{\varepsilon} \mu \varepsilon v$ AN[ .......... . . .

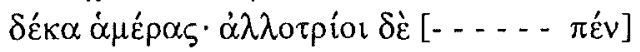

$\tau \varepsilon \dot{\alpha} \mu \dot{\varepsilon} \rho \alpha \varsigma \cdot \tau \grave{\alpha} \delta \dot{\varepsilon} \lambda \lambda_{0} \pi \dot{\alpha} \mathrm{EY}[\ldots . . . .$.

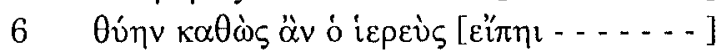

vacat 
c) Chapelle de Aghios Nicolas (Aï-Nicolas), au nord et en contrebas de la colline du sanctuaire, à $c$. $30 \mathrm{~m}$. au-delà de la route municipale conduisant au village moderne de Lykosoura : l'autel de la chapelle est formé d'un chapiteau dorique bien conservé $(88 \mathrm{~cm} \mathrm{x} \mathrm{c.} 96 \mathrm{~cm})$ reposant sur une colonnette (h. : $60 \mathrm{~cm})$; le pupitre de la chapelle est formé d'une partie de colonnette (h. : $100 \mathrm{~cm}$, d. : $42 \mathrm{~cm}$ ); dans le mur sud sont insérées deux colonnettes; devant l'entrée gisent trois fragments de colonnes non cannelées et sur l'une d'elles repose une plaque en marbre; sur le sol, à côté du mur nord, gisent deux colonnes sans cannelures (l. : 150 et $102 \mathrm{~cm}$, d. : c. $53 \mathrm{~cm}$ ).

d) Parmi les blocs de pierres formant les murs des chapelles cidessus, un bon nombre doit provenir du site antique.

\section{Prospection archéologique de surface}

L'étude du matériel étant en cours, nous nous limiterons à quelques remarques.

Dans toute la région indiquée sur la carte (fig. 5), le sol contient des dizaines de morceaux de briques et de tessons de céramique tardive au $\mathrm{m}^{2}$. Des tranchées effectuées à une profondeur de $c .15 \mathrm{~cm}$ de la surface du sol aux endroits indiqués par un $X$ sur la carte (fig. 5) ont révélé une situation analogue, mais avec un nombre important de tessons de céramique hellénistique et classique et, dans une moindre mesure, archaïque. Des tessons géométriques n'ont pas été repérés, mais quelques-uns pourraient bien dater de l'époque mycénienne.

Selon le plan du travail, la recherche reprendra vers la fin juillet 1991 et sera fixée sur une prospection de la surface du sol, encore plus systématique, accompagnée éventuellement de tranchées limitées, ainsi que sur l'étude topographique générale de la région, afin que des fouilles systématiques puissent commencer en 1992 ou en 1993. Les chercheurs désirant participer à ces activités du C.E.R.G.A. peuvent introduire une demande jusqu'au 20 mai 1991 à : R. Laffineur, Comité de recherches scientifiques du C.E.R.G.A., Service d'archéologie grecque, Université de Liège, 32 place du XX-Août, B - 4000 Liège (Belgique), ou à I. Loucas, Centre Culturel Européen de Delphes, Karneadou 13, GR - 10675 Athènes (Grèce) (fax n 7230676).

I. LOUCAS \& É. LOUCAS-DURIE 


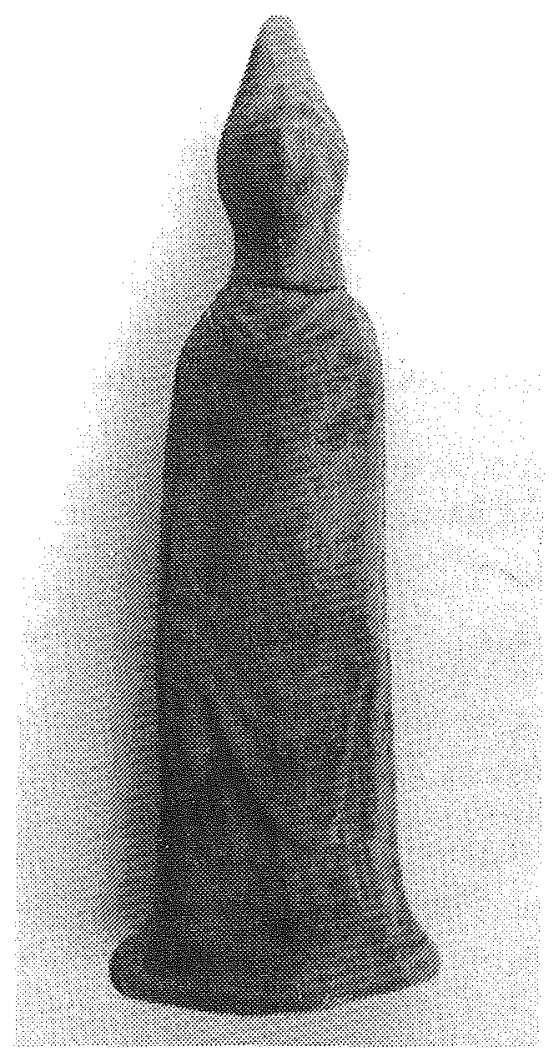

Fig. 1 : Statuette de femme (terre cuite)

Fig. 2 : Lampes
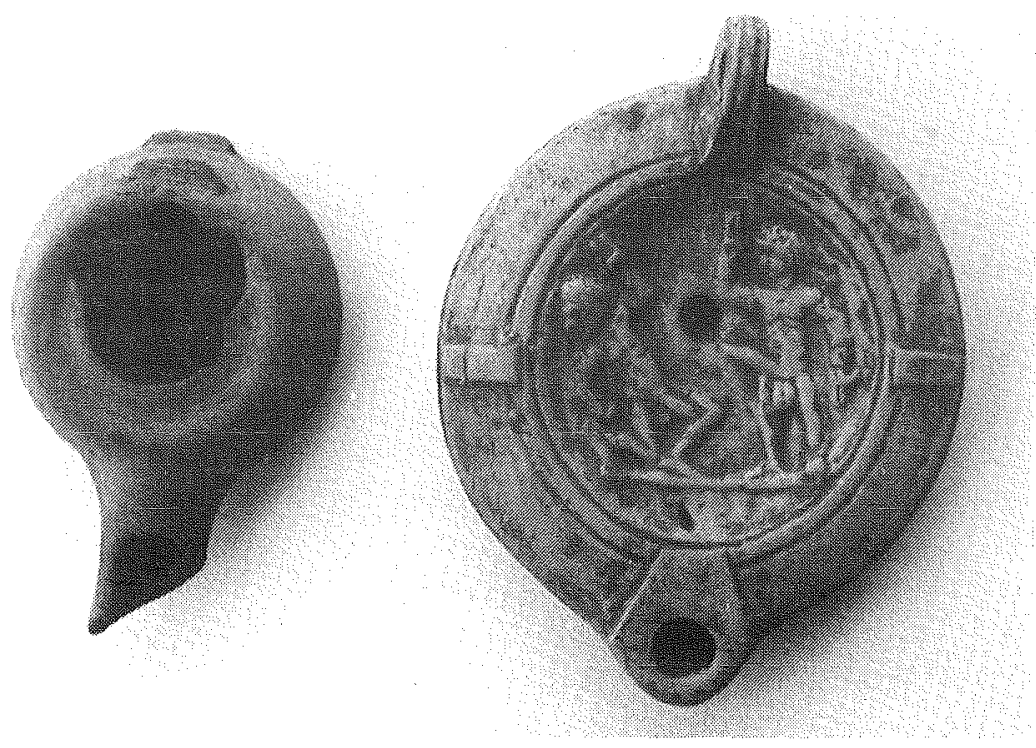

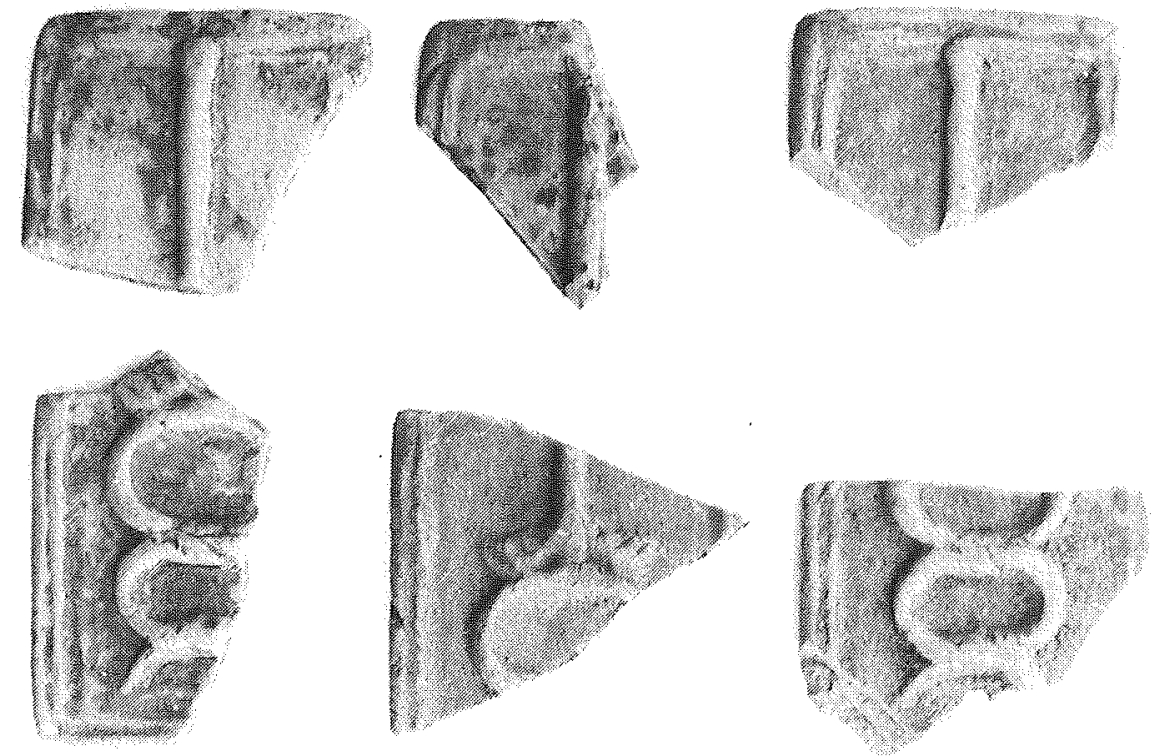

Fig. 3 : Plaques à motifs de caducée (terre cuite)

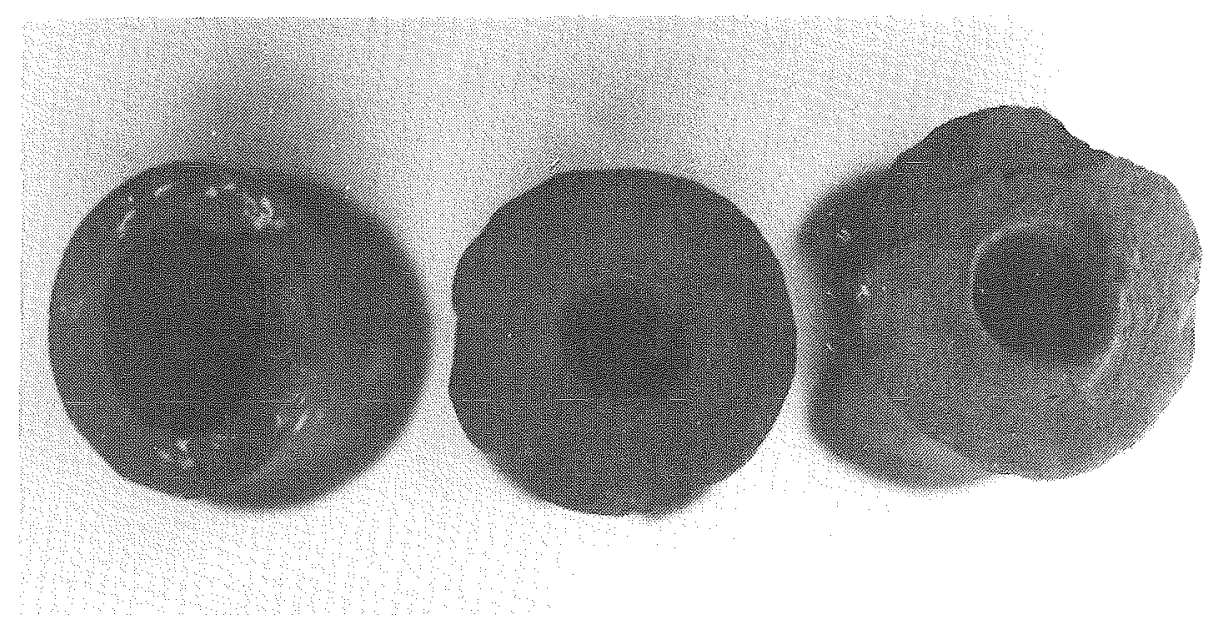

Fig. 4 : Vases à libation (?) : vue d'en haut de la base 


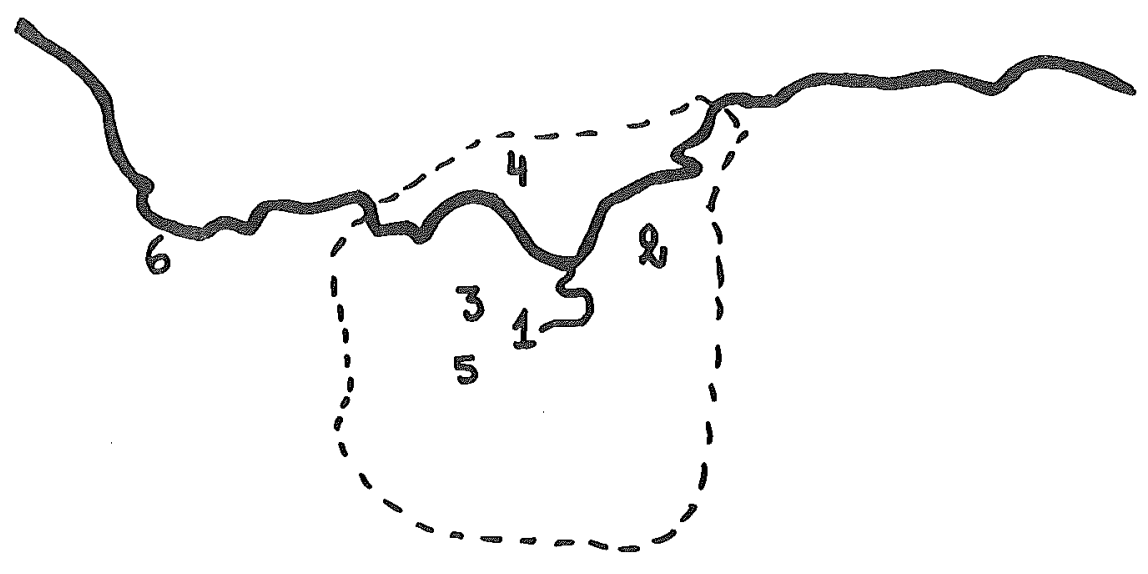

Fig. 5 : Carte 1. Sanctuaire de Despoina 2. Chapelle de Aghios Helias

3. Chapelle de Aghios Georghios

4. Chapelle de Aghios Nicolaos

5. Chapelle de Aghia Heleni

6. Village actuel (Lykosoura)

7. Village Petrovouni 